\title{
Novel Dextransucrase Gtf-DSM, Highly Similar in Sequence to Reuteransucrase GtfO, Displays Unique Product Specificity
}

Ziwei Chen, ${ }^{\dagger}$ Yuqing Tian, ${ }^{\dagger}$ Wenli Zhang, ${ }^{\dagger}$ Cuie Guang, ${ }^{\dagger}$ Xiangfeng Meng ${ }^{*} \star \star$ and

$$
\text { Wanmeng } \mathrm{Mu}^{*}, \dagger, \S
$$

${ }^{\dagger}$ State Key Laboratory of Food Science and Technology and $\$$ International Joint Laboratory on Food Safety, Jiangnan University, Wuxi, Jiangsu, 214122, China.

${ }^{\S}$ State Key Laboratory of Microbial Technology, Shandong University, Qingdao, Shandong, 266237, China.

* Corresponding author.

E-mail address: wmmu@jiangnan.edu.cn. (W.M.), x.meng@sdu.edu.cn (X.M.). 
Figure S1. The SEC profile of recombinant Gtf-DSM. The main peak ranging from 68 to 78 min corresponds to a monomeric protein according to the elution time and determined standard proteins. The target protein was collected within this main peak.

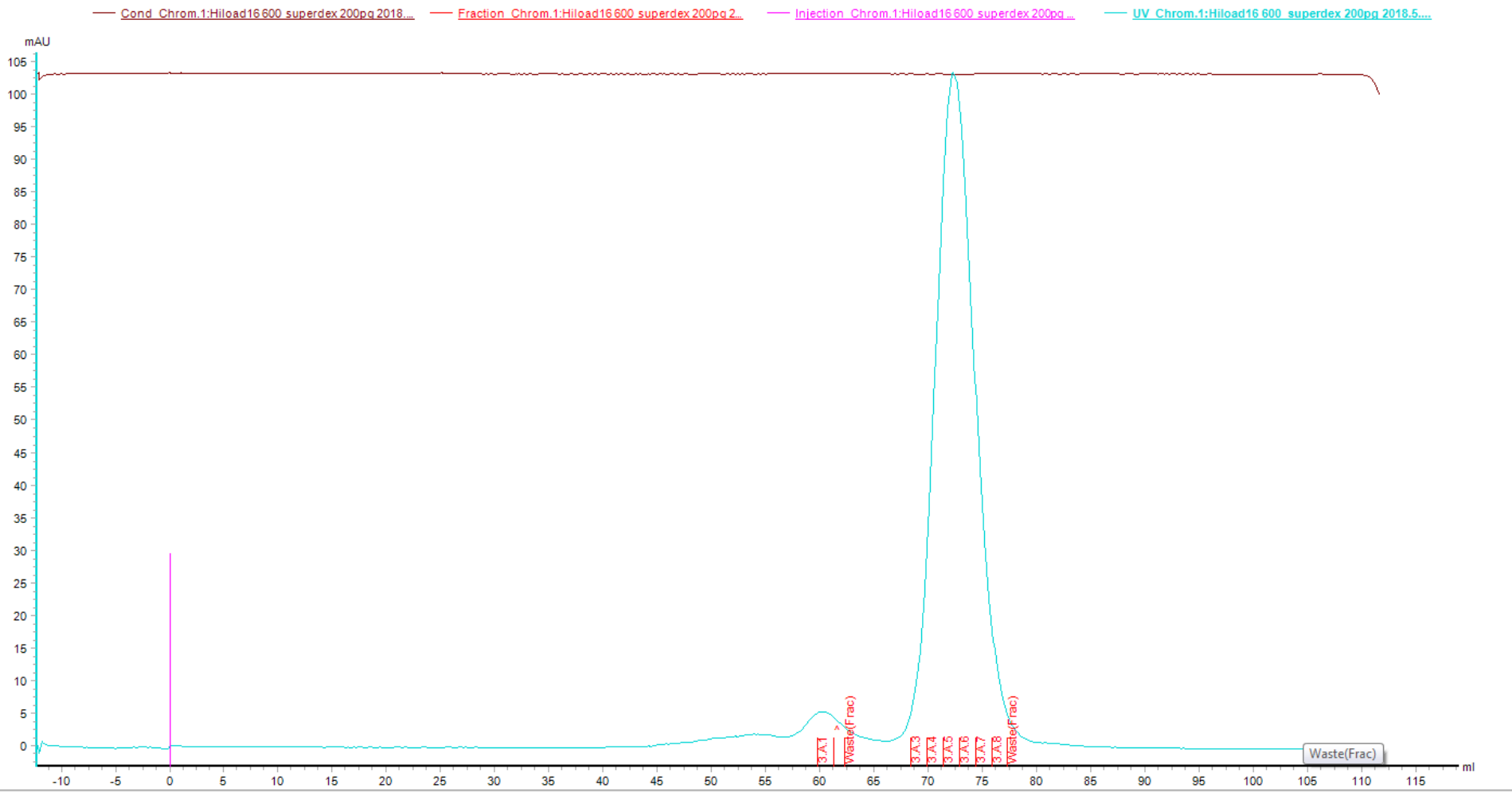


Figure S2. Amino acid sequence alignment of conserved motifs I (green), II (raspberry), III (blue) and IV (yellow) of Gtf-DSM and reported GS enzymes. The strictly conserved residues are highlighted by a red background. The acceptor binding subsites $-1,+1$ and +2 of the resolved Gtf180 enzyme are correspondingly labelled at the bottom of motifs. NU, nucleophile; A/B, general acid/base; TS, transition state stabilizer.

\begin{tabular}{|c|c|}
\hline GSs & Motif I \\
\hline Gtf-DSM & 1508 ADWVP DQ \\
\hline Gtf180 & $A D W V P D$ \\
\hline GtfA & ADWVPD \\
\hline GtfO & $A D W V P D$ \\
\hline GtfML 1 & $A D W V P I$ \\
\hline GtfKg15 & $\mathrm{ADFVP}$ \\
\hline GtfKg3 & $A D F V P$ \\
\hline Gtf33 & DDWVP \\
\hline DSRS & $A D W V P$ \\
\hline DSRBCB4 & $A D W V P$ \\
\hline DSR-A & $\mathrm{NDWVP}$ \\
\hline DSR-B & $\mathrm{ADWVP}$ \\
\hline DSR-DP & $A D Y V P$ \\
\hline DSR-M & $\mathrm{ADFVP}$ \\
\hline BRS-A & $\mathrm{A} D \mathrm{~V} \boldsymbol{V} \mathrm{A}$ \\
\hline DSR-E-CD2 & ADVVD \\
\hline ASR & ADWVP \\
\hline BRS-B & $\mathrm{ADF} \mathrm{VA}$ \\
\hline BRS-C & $\mathrm{ADY} \mathbf{V} \mathrm{A}$ \\
\hline DSR-F & $A D W V P$ \\
\hline DSRI & ADWVP \\
\hline GtfB-GS5 & $A D W V P$ \\
\hline GtfC-GS5 & ADWVP \\
\hline GtfB-MT8148 & $\mathrm{ADWVP}$ \\
\hline GtfC-MT8148 & $A D W V P$ \\
\hline GtfD-MT8148 & $\mathrm{VVP}$ \\
\hline GtfR & VP \\
\hline DSRWC & $\mathrm{ADF} \mathbf{V}$ \\
\hline
\end{tabular}

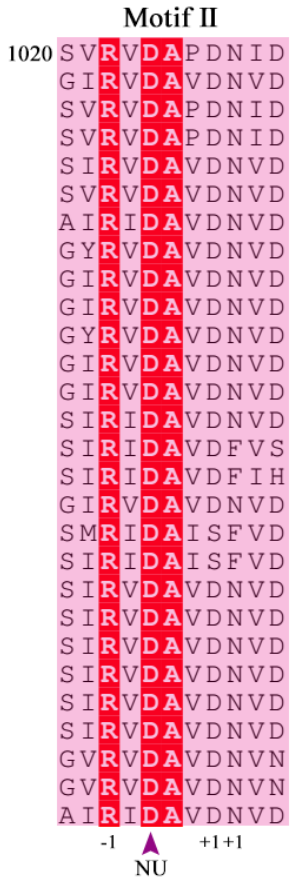

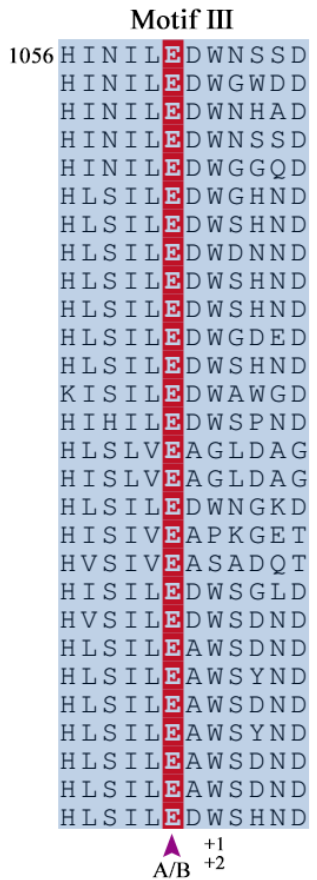

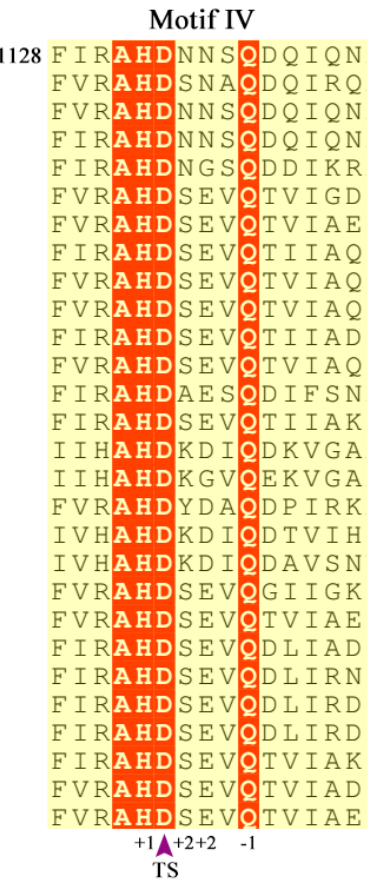


Figure S3. Amino acid sequence alignment of twelve residues of native Gtf-DSM with that of other reported GS enzymes.

\begin{tabular}{|c|c|c|c|c|c|c|c|c|c|c|c|c|}
\hline & 739 & 758 & 765 & 768 & 889 & 937 & 977 & 1083 & 1086 & 1087 & 1458 & 1529 \\
\hline GTF-DSM & L & V & $Q$ & D & S & I & D & D & $Q$ & $\mathrm{~K}$ & D & V \\
\hline GTFO & $\mathrm{F}$ & G & $\bar{H}$ & $Y$ & $\mathrm{~N}$ & L & A & V & $\bar{K}$ & G & A & G \\
\hline GTF180 & $\mathrm{F}$ & $\mathrm{P}$ & $Q$ & $\mathrm{~N}$ & $\mathrm{~N}$ & $\mathrm{~L}$ & A & D & $\mathrm{R}$ & $\mathrm{N}$ & D & $\mathrm{S}$ \\
\hline GTFA & $\mathrm{E}$ & $\mathrm{P}$ & $Q$ & $\mathrm{~N}$ & $\mathrm{~N}$ & I & $Q$ & D & K & $\mathrm{N}$ & D & V \\
\hline GTFML1 & $\mathrm{E}$ & $\mathrm{P}$ & $Q$ & $\mathrm{~N}$ & $\mathrm{~N}$ & L & $\mathrm{S}$ & $Y$ & $\mathrm{~S}$ & $\mathrm{~T}$ & D & V \\
\hline GTFKg15 & $\mathrm{T}$ & L & I & $\mathrm{N}$ & $\mathrm{N}$ & D & $Y$ & D & $\mathrm{H}$ & $\mathrm{T}$ & D & $\mathrm{T}$ \\
\hline GTFKg3 & $\mathrm{T}$ & $\mathrm{M}$ & S & D & $\mathrm{T}$. & D & Y & D & $\mathrm{H}$ & $\mathrm{T}$ & D & $\mathrm{T}$ \\
\hline GTF33 & S & V & $I$ & G & $\mathrm{T}$ & D & $Y$ & $\mathrm{~F}$ & $\mathrm{H}$ & L & D & $\mathrm{T}$ \\
\hline DSRS & $\mathrm{T}$ & L & V & G & $\mathrm{N}$ & D & $\mathrm{E}$ & D & $\mathrm{H}$ & $\mathrm{T}$ & D & $\mathrm{T}$ \\
\hline DSRBCB 4 & I & L & V & G & $\mathrm{N}$ & D & $\mathrm{F}$ & D & $\mathrm{H}$ & $\mathrm{T}$ & D & $\mathrm{T}$ \\
\hline DSR-A & $\mathrm{P}$ & $\mathrm{P}$ & I & G & $\mathrm{T}$ & D & $\mathrm{F}$ & $\mathrm{E}$ & $\mathrm{H}$ & L & D & $\mathrm{T}$ \\
\hline DSR-B & $I$ & L & V & G & $\mathrm{N}$ & D & $\mathrm{E}$ & $\mathrm{D}$ & $\mathrm{H}$ & $\mathrm{T}$ & D & $\mathrm{T}$ \\
\hline DSR-DP & $\mathrm{T}$ & L & $I$ & G & $\mathrm{L}$ & D & $Y$ & $\mathrm{~T}$ & K & D & D & V \\
\hline DSR-M & $\mathrm{T}$ & $\mathrm{R}$ & V & $\mathrm{N}$ & $\mathrm{N}$ & $\mathrm{N}$ & $Y$ & A & $Q$ & $\mathrm{~N}$ & D & $\mathrm{T}$ \\
\hline BRS-A & K & $\mathrm{Q}$ & $\mathrm{N}$ & G & $\mathrm{N}$ & . & L & K & $\mathrm{T}$ & $\mathrm{N}$ & $\mathrm{D}$ & V \\
\hline DSR-E-CD2 & K & $Q$ & $I$ & G & $\mathrm{N}$ & A & $\mathrm{E}$ & $\mathrm{L}$ & $\mathrm{T}$ & $\mathrm{N}$ & D & A \\
\hline ASR & $\mathrm{T}$ & L & $\mathrm{T}$ & $\mathrm{N}$ & $\mathrm{N}$ & $Q$ & $\mathrm{~S}$ & $Y$ & $\mathrm{~T}$ & $\mathrm{~S}$ & D & V \\
\hline BRS-B & K & $\mathrm{Q}$ & $\mathrm{N}$ & G & $\mathrm{N}$ & . & $\mathrm{L}$ & $Q$ & S & K & G & V \\
\hline BRS-C & K & $Q$ & $\mathrm{~N}$ & G & $\mathrm{N}$ & . & M & $\mathrm{N}$ & S & K & G & V \\
\hline DSR-F & K & L & $\mathrm{T}$ & $\mathrm{N}$ & $\mathrm{N}$ & $Q$ & S & $\mathrm{T}$ & $Q$ & $\mathrm{~N}$ & D & V \\
\hline DSRI & K & V & V & G & $\mathrm{N}$ & D & $Y$ & $\mathrm{~N}$ & $\mathrm{R}$ & L & $\mathrm{D}$ & V \\
\hline GTFB-GS5 & $\mathrm{P}$ & V & I & G & $\mathrm{N}$ & D & $Y$ & $\mathrm{~N}$ & $\mathrm{R}$ & L & D & V \\
\hline GTFC-GS5 & K & L & $\mathrm{N}$ & G & $\mathrm{N}$ & D & Y & $\mathrm{N}$ & $\mathrm{R}$ & L & D & V \\
\hline GTFB-MT8148 & $\mathrm{P}$ & V & I & G & $\mathrm{N}$ & D & $Y$ & $\mathrm{~N}$ & $\mathrm{R}$ & L & D & V \\
\hline GTFC-MT8148 & K & L & $\mathrm{N}$ & G & $\mathrm{N}$ & D & $\mathrm{Y}$ & $\mathrm{N}$ & $\mathrm{R}$ & $\mathrm{L}$ & D & V \\
\hline GTFD-MT8148 & $\mathrm{P}$ & $\mathrm{P}$ & $\mathrm{T}$ & $\mathrm{N}$ & $\mathrm{N}$ & D & Y & $\mathrm{N}$ & $\mathrm{R}$ & L & D & V \\
\hline GTFR & S & V & $E$ & G & $\mathrm{T}$ & D & Y & $\mathrm{N}$ & $\mathrm{R}$ & L & $\mathrm{D}$ & V \\
\hline DSRWC & $\mathrm{T}$ & M & S & D & $\mathrm{T}$. & D & $Y$ & D & $\mathrm{H}$ & $\mathrm{T}$ & D & $\mathrm{T}$ \\
\hline & N-ter & \multicolumn{2}{|c|}{ Domain V } & \multicolumn{4}{|c|}{ Domain B } & \multicolumn{4}{|c|}{ Domain A } & Domain B \\
\hline
\end{tabular}


Figure S4. Nano DSC analysis of the recombinant Gtf-DSM.

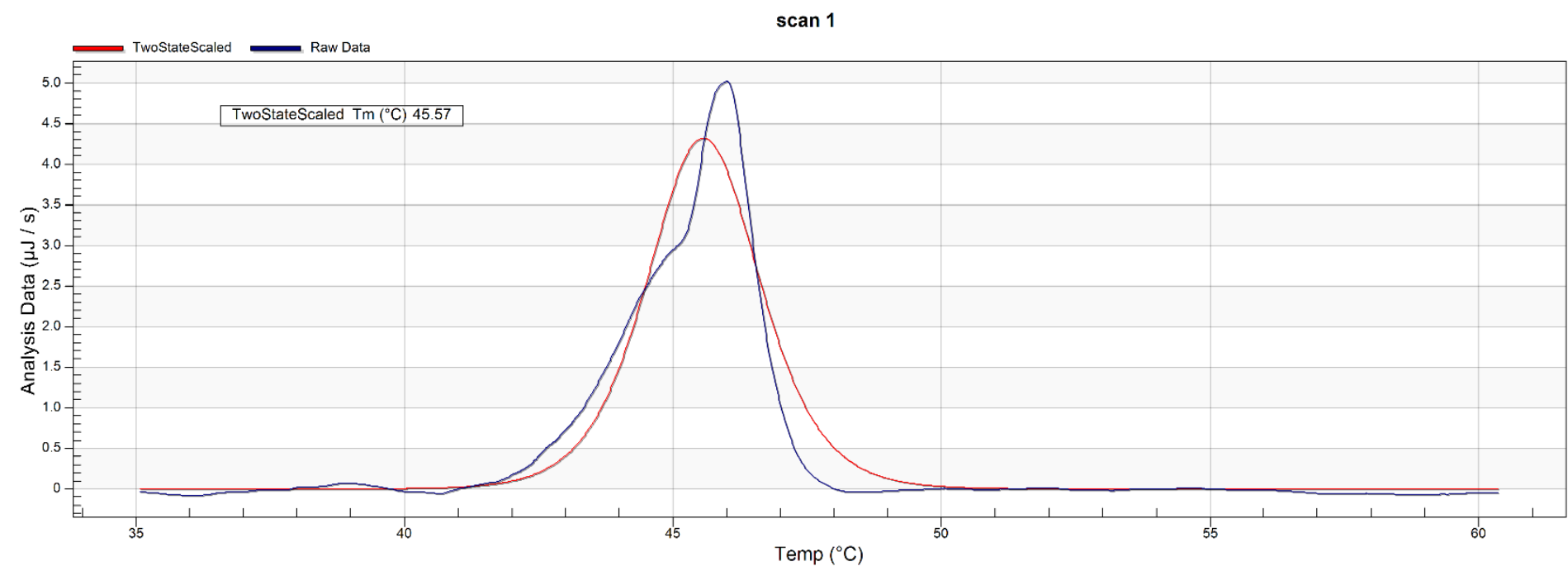


Figure S5. The HPSEC analysis with RI and MALLS detectors for the dextran produced by the recombinant Gtf-DSM. The differential refractive index and molecular weight distribution were represented by blue and red lines respectively.

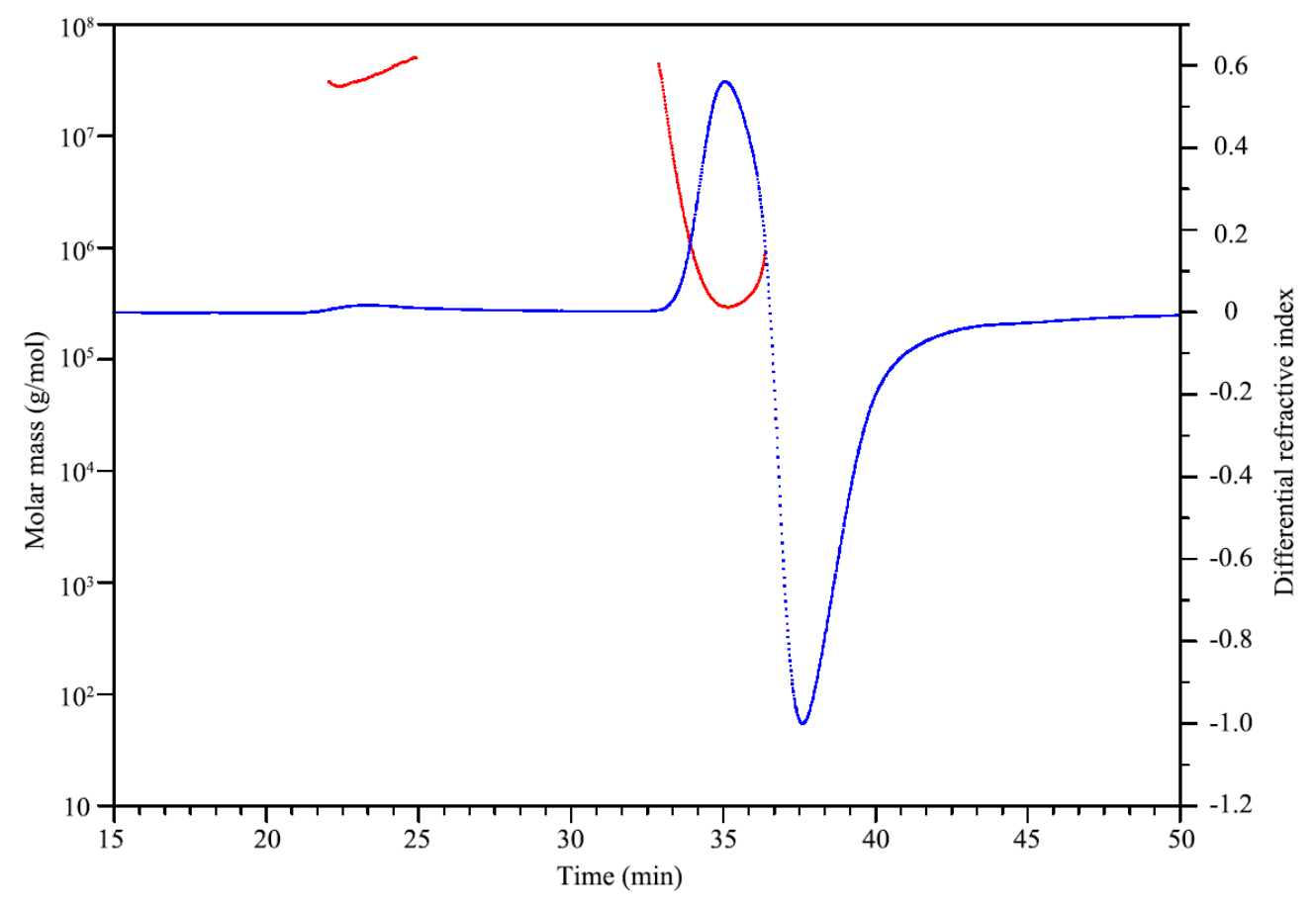


Table S1. Comparison of amino acid sequence homology of the Gtf-DSM and reported GS enzymes from various microorganisms. ${ }^{\text {a }}$

\begin{tabular}{cc}
\hline GS enzymes & Identity $(\%)$ \\
\hline Gtf-DSM & 100 \\
Gtf180 & 60.91 \\
GtfA & 69.72 \\
GtfO & 99.33 \\
GtfML1 & 58.12 \\
GtfKg15 & 40.49 \\
GtfKg3 & 42.18 \\
Gtf33 & 43.64 \\
DSRS & 43.55 \\
DSRBCB4 & 42.11 \\
DSR-A & 45.16 \\
DSR-B & 44.38 \\
DSR-DP & 37.66 \\
DSR-M & 38.88 \\
BRS-A & 40.3 \\
DSR-E-CD2 & 45.39 \\
ASR & 41.72 \\
BRS-B & 38.07 \\
BRS-C & 37.42 \\
DSR-F & 42.11 \\
DSRI & 43.91 \\
GtfB-GS5 & 44.04 \\
GtfC-GS5 & 43.87 \\
GtfB-MT8148_Gtf-I_ & 44.64 \\
GtfC-MT8148_Gtf-SI_ & 42.36 \\
GtfD-MT8148_Gtf-S_- & 42.58 \\
GtfR & 40.09 \\
DSRWC & 42.23 \\
\hline GtfKg15 (Lactobacilus & GtKei Ko15 \\
\hline
\end{tabular}

${ }^{a} \mathrm{Gtf} 180$ (L. reuteri 180), GtfKg15 (Lactobacillus sakei Kg15), GtfKg3 (Lactobacillus fermentum Kg3), Gtf33 (Lactobacillus parabuchneri 33), DSRS (L. mesenteroides NRRL B-512F), DSRBCB4 (L. mesenteroides B-1299 CB4), DSR-A (L. citreum NRRL B-1299), DSR-B (L. citreum NRRL B1299), DSR-DP (L. citreum NRRL B-1299), DSR-M (L. citreum NRRL B-1299), DSR-F ( $L$. citreum B/110-1-2), DSRWC (Weissella cibaria CMU), GtfR (Streptococcus oralis ATCC10557), GtfA (L. reuteri 121), GtfO (L. reuteri ATCC 55730), GtfML1 (L. reuteri ML1), DSRI ( $L$. mesenteroides NRRL B-1118), GtfB-GS5 (S. mutans GS5), GtfC-GS5 (S. mutans GS5), GtfBMT8148 ( S. mutans MT8148), GtfC-MT8148 ( S. mutans MT8148), GtfD-MT8148 ( S. mutans MT8148), ASR (L. mesenteroides NRRL B-1355), DSR-E CD2 (L. citreum NRRL B-1299), BRSA (L. citreum NRRL B-1299), BRS-B (L. citreum NRRL B-742), BRS-C (L. fallax KCTC3537). 